\title{
Wearable devices for remote vital signs monitoring in the outpatient setting: an overview of the field
}

\author{
Stephanie Soon, ${ }^{1}$ Hafdis Svavarsdottir, ${ }^{2}$ Candice Downey (D) , \\ David George Jayne ${ }^{2}$
}

${ }^{1}$ School of Medicine, University of Leeds, Leeds, UK

'Leeds Institute of Medical Research at St. James's, St. James's University Hospital, University of Leeds, Leeds, UK

\section{Correspondence to}

Candice Downey, Leeds Institute of Medical Research at St. James's, University of Leeds, Leeds, LS2 9JT, UK; c.l.downey@ leeds.ac.uk

The findings of this review have been presented as a poster at the National Student Association for Medical Research (NSAMR) Conference in January 2019, and at the Association of Surgeons in Training International Surgical Conference in March 2019. The abstract will be published in the British Journal of Surgery.

Received 5 April 2019 Revised 13 September 2019 Accepted 13 December 2019 Published Online First 14 January 2020

\section{Check for updates}

(C) Author(s) (or their employer(s)) 2020. No commercial re-use. See rights and permissions. Published by BMJ.

\footnotetext{
To cite: Soon S, Svavarsdottir H, Downey C, et al. BMJ Innov 2020;6:5571.
}

\begin{abstract}
Early detection of physiological deterioration has been shown to improve patient outcomes. Due to recent improvements in technology, comprehensive outpatient vital signs monitoring is now possible. This is the first review to collate information on all wearable devices on the market for outpatient physiological monitoring. A scoping review was undertaken. The monitors reviewed were limited to those that can function in the outpatient setting with minimal restrictions on the patient's normal lifestyle, while measuring any or all of the vital signs: heart rate, ECG, oxygen saturation, respiration rate, blood pressure and temperature.

A total of 270 papers were included in the review. Thirty wearable monitors were examined: 6 patches, 3 clothing-based monitors, 4 chest straps, 2 upper arm bands and 15 wristbands. The monitoring of vital signs in the outpatient setting is a developing field with differing levels of evidence for each monitor. The most common clinical application was heart rate monitoring. Blood pressure and oxygen saturation measurements were the least common applications. There is a need for clinical validation studies in the outpatient setting to prove the potential of many of the monitors identified.

Research in this area is in its infancy. Future research should look at aggregating the results of validity and reliability and patient outcome studies for each monitor and between different devices. This would provide a more holistic overview of the potential for the clinical use of each device.
\end{abstract}

\section{INTRODUCTION}

Regular vital signs monitoring is a common inpatient care intervention, which aims to facilitate the early recognition of abnormal physiological parameters in deteriorating patients. Derangements in vital signs is a known predictor of cardiac and respiratory arrest, ${ }^{1}$ and early detection of deterioration has been shown to improve patient outcomes including mortality and quality of life. ${ }^{2}$ Traditional intermittent manual vital signs monitoring, such as early warning score systems, risks undetected patient deterioration through inadequate frequency of monitoring. ${ }^{3}$ Wearable remote monitoring technologies, aided by wireless data transmission, allow continuous monitoring of patients' vital signs and introduce the possibility of physiological monitoring in the outpatient setting.

Until recently, outpatient vital signs monitoring has been mostly limited to ECG monitoring with Holter devices. These monitors have been used for over 40 years as a non-invasive method of continuously monitoring heart rate and ECG for set periods of time. ${ }^{4-6}$ Holter monitors do not allow real-time monitoring or monitoring of other vital signs such as oxygen saturation, blood pressure, respiratory rate and temperature. ${ }^{7}$ Recent improvements in battery technology and wireless data transmission alongside the advent of smartphones have heralded advances in wearable monitors. In the last 15 years, wearable monitors have been developed that incorporate multiple sensors, intelligent processing, alarms to support medical decisions and interactions with the health provider.

There are a number of wearable remote monitoring systems available. The level of evidence to support these systems is variable and the evaluation of remote wearable monitoring systems has been largely limited to the inpatient setting. As overall healthcare burden increases and scarcity of hospital beds leads to accelerated discharges, there is increasing interest in 
the application of remote monitoring in the outpatient setting. ${ }^{8}$

With increased interest in the use of remote outpatient monitoring, there is a clear need for a collective analysis of the efficacy of currently available wearable remote monitoring systems. At present, the only available review of remote monitoring systems applies to the inpatient setting and focuses solely on the accelerometer functions of wearable devices. ${ }^{9}$ This is the first review to compile the evidence for wearable devices for outpatient physiological monitoring. This study aims to provide a comprehensive overview of currently available systems and to evaluate and synthesise the evidence for each system to identify areas that require further evaluation.

\section{METHODS}

\section{Study design}

The study has been conducted in the form of a scoping review to map all the existing literature on the topic to allow a broad overview of the area and identification of gaps in the evidence.

\section{Inclusion criteria}

Studies were selected according to the criteria outlined below:

\section{Types of studies}

Included studies reported evidence on outpatient wearable vital signs monitors. Peer-reviewed articles, trial registrations and grey literature such as white papers were included. As many of the wearable devices are in the early stages of evaluation, confining the search to peer-reviewed publications was not appropriate.

\section{Types of participants}

Studies were limited to those involving outpatients.

Types of interventions/comparators

The monitors under review were limited to those that could function in the outpatient setting with minimal restrictions to the patient's normal lifestyle, while measuring any or all of the vital signs: heart rate, ECG, oxygen saturation, respiration rate, blood pressure and temperature. Only monitors that allow real-time monitoring were included.

Types of outcome measures

The selection of studies was not limited by the outcome measures reported. Evidence included reliability studies, evaluations of patient perspectives, clinical evaluations reporting patient outcomes and studies where the device was used in a trial environment but not tested in itself. Outcomes were collected as reported in the individual studies.

\section{Exclusion criteria}

Studies were excluded if they reported only the development of the technology for such monitors. Studies

\section{Box 1 The initial search criteria}

Initial search strategy keywords

- Vital Signs AND Outpatient Monitor* OR

- Vital Signs AND Monitor* OR

- Vital Signs AND Outpatient OR

- Fitness AND Monitor

were excluded if they reviewed monitors that were no longer in production or those that were not wearable or functional only in the inpatient setting.

\section{Information sources}

Electronic searches

The search was conducted in two stages. MEDLINE, ClinicalTrials.gov, NICE.org, Google and PubMed Central (PMC) were searched for articles published from 1996 to June 2018 using the search terms outlined in box 1 . Once the monitors were identified, a second search was undertaken; table 1 outlines the search terms and strategy used in the second search.

\section{Searching other resources}

References and citations of selected studies were searched to ensure completeness. Company websites for each of the products were also searched for white papers and links to peer-reviewed publications.

\section{Data collection and analysis}

Selection of studies

Studies were initially screened by title and abstract followed by a full text review. Studies for screening were recorded in a Microsoft Word document (Microsoft Word for Mac 2011, V.14.3.9, Microsoft, USA).

\begin{tabular}{|c|c|}
\hline \multicolumn{2}{|c|}{ Final search terms used (individually) } \\
\hline Vital Connect Patch & Accurate24 \\
\hline BodyGuardian Heart & Vincense WHMS \\
\hline Sensium Vitals System & SpryHealth Loop \\
\hline Intelesens Zensor & VisiMobile \\
\hline SEEQ MCT & Helo Lx \\
\hline Nuvant MCT & FitBit \\
\hline Kenzen Patch & Fitbit and Heart Rate Monitor \\
\hline OmBra & Fitbit Charge and Heart Rate Monitor \\
\hline OmSignal & Apple Watch \\
\hline Nuubo & Apple Watch and Heart Rate Monitor \\
\hline Hexoskin & Garmin Vivofit \\
\hline QardioCore & Garmin Vivosmart \\
\hline EQ02 Lifemonitor & Samsung Gear 2 \\
\hline Zephyr BioHarness 3 & Xiao Mi Band \\
\hline Polar H7 & Empatica E4 \\
\hline Polar H7 Vital Sign & Epson Pulse Sense Watch \\
\hline Polar H7 Monitor & Microsoft Band 2 \\
\hline Everion & Striiv Fusion Bio 2 \\
\hline Snap40 & \\
\hline
\end{tabular}


Selected studies were collected and stored on Mendeley (V.1.19.2, Elsevier, USA).

\section{Data extraction}

Data were compiled in a table in Microsoft Word. This included information on the trade name, type of monitor, vital sign(s) measured and level of evidence.

\section{Data synthesis}

A narrative synthesis approach was used to summarise study findings. Monitors were grouped according to the type of device. Commonalities were sought in the type and volume of evidence substantiating each monitor. This evidence was then synthesised to assess the gaps in the literature for each monitor. Quantitative data synthesis was avoided due to the heterogeneity of outcome measures reported by selected studies.
Assessment of risk of bias in included studies

Due to the heterogeneity of the included study types and the preponderance of grey literature a formal assessment of risk of bias was not undertaken.

\section{RESULTS}

In total, 270 papers were identified that met the eligibility criteria. Figure 1 illustrates the selection process. Thirty wearable monitors were examined: 6 patches, 3 clothingbased monitors, 4 chest straps, 2 upper arm bands and 15 wristbands. Examples of each of these devices are illustrated in figure 2. The monitoring of vital signs in the outpatient setting is a developing field with differing levels of evidence for each monitor. The evidence base for each monitor is summarised in tables 2-7.

\section{Patch monitors}

The review identified six wireless monitoring patches. All of the patch monitors measure heart rate and
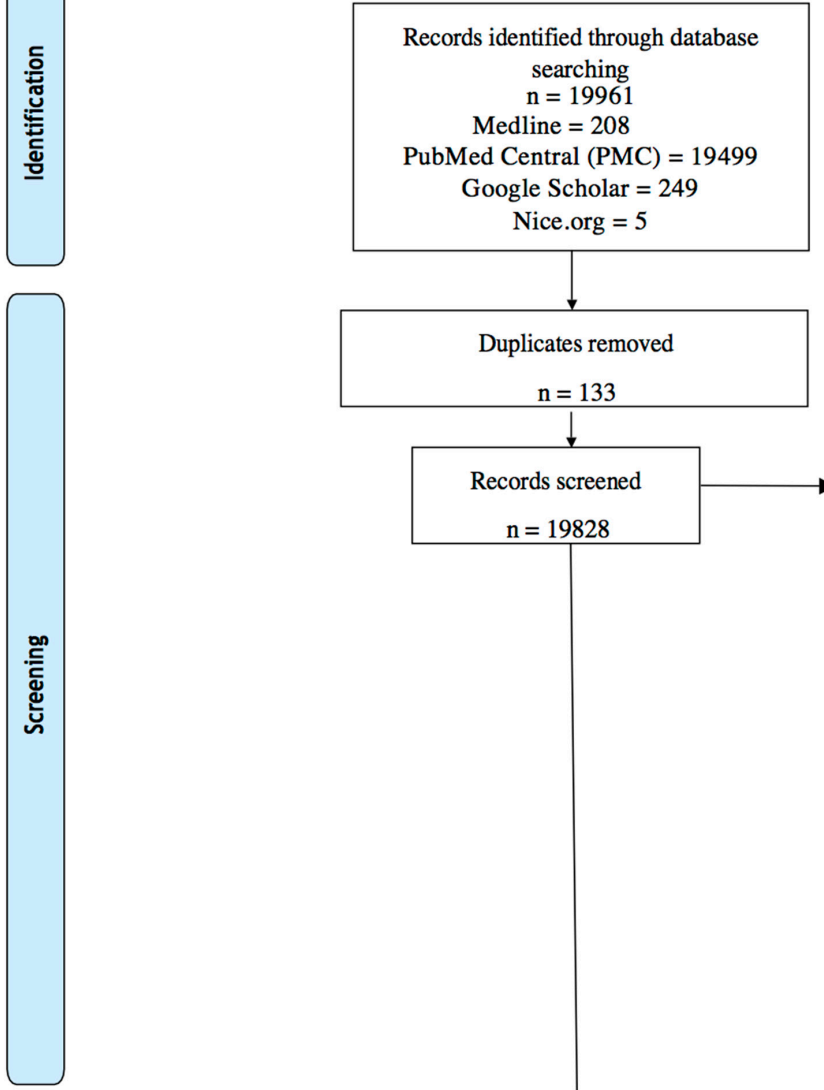

Records excluded from title, abstract and full text articles $n$ $=19558$

- (Non English Articles $=3$

- Monitors not measuring vital signs $=4820$

- Studies not mentioning vital signs $=1830$

- Non wearable monitors $=5929$

- Not applicable to outpatient setting $=$ 6830

- Studies describing the development of monitoring technology $=57$

- Monitors nolonger in production $=89$ )

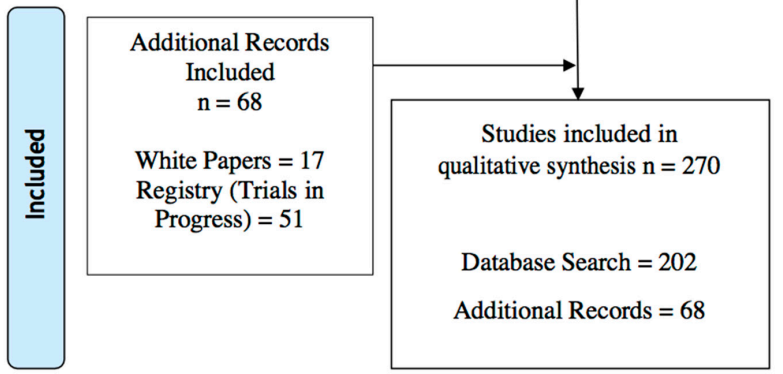

Figure 1 PRISMA diagram of search and eligibility process 


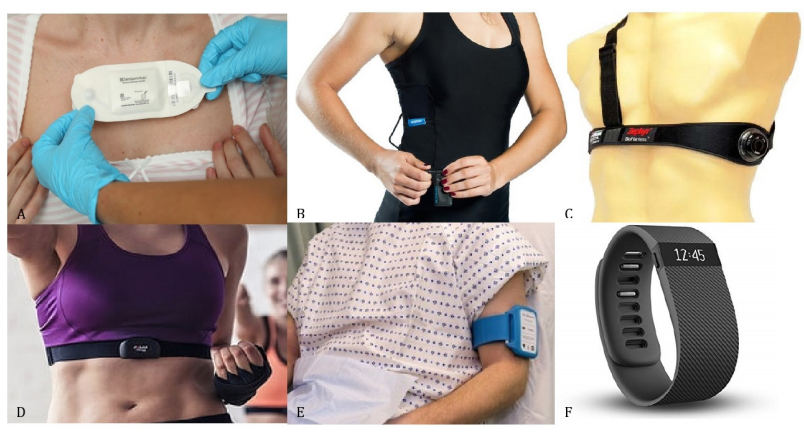

Figure 2 Examples of outpatient monitoring devices included in this study. (A) Medical Grade Adhesive Patch: from Sensium, Abingdon, UK. (B) Clothing with embedded sensors: from Hexoskin, Montreal, Canada. (C) Chest strap: from Medtronic, Maryland, USA. (D) Chest strap: from Polar Electro, Warwick, UK. (E) Upper armband: from Current, Edinburgh, UK. (F) Wristband: from Fitbit, San Francisco, USA.

adhere to the patient with disposable stickers. Table 2 summarises the vital signs recorded by and the current evidence available for each device.

The patch with the most evidence was the SensiumVitals system, a patch monitoring heart rate, respiratory rate and temperature. Nine of its 17 total publications were case studies evaluating the use of the SensiumVitals system in a variety of clinical contexts from diagnosing meningitis ${ }^{10}$ to detecting opioidinduced respiratory depression. The authors conclude that the patch can feasibly be used in these contexts given its high levels of accuracy and patient compliance. However, it should be noted that these papers were written and published by Sensium Case Studies, a subgroup of the company producing the SensiumVitals patch.

Five of the six patches examined have validity or reliability studies. Of these, two have validity data but have not been tested in a clinical setting with no patient outcome studies. This may be due to the developing nature of the patch market with many patches in the early stages of product release.

For example, the patch with the least evidence was the Kenzen Patch with zero peer-reviewed publications and grey literature; it currently also lacks FDA certification. The product works differently from the other mentioned patches by analysing the biomarkers available in an individual's sweat such as sodium and glucose in addition to measuring heart rate and temperature. Currently being marketed at athletes for its sweat-analysing biosensor, several American sports teams such as the San Francisco 49ers have signed up to trial this patch.

\section{Clothing monitors}

The three clothing-based monitors examined in this study function via monitors embedded in wearable garments. These items include a sports bra (OmBra), sports shirt (Hexoskin) and vest to be worn under

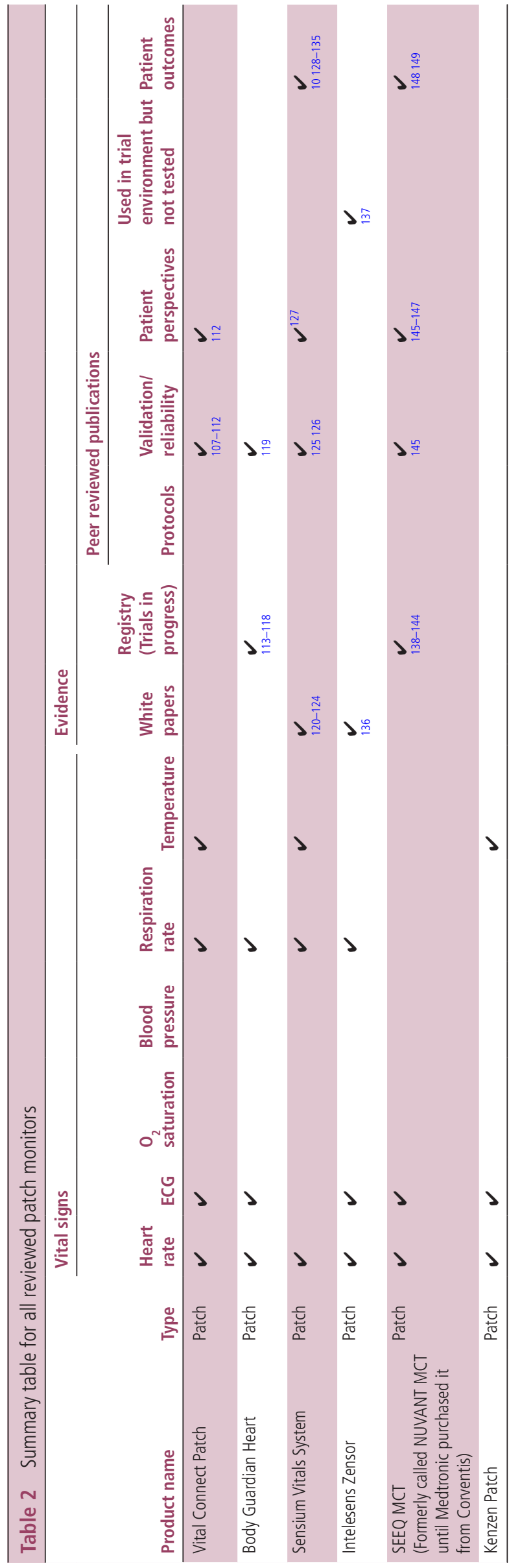



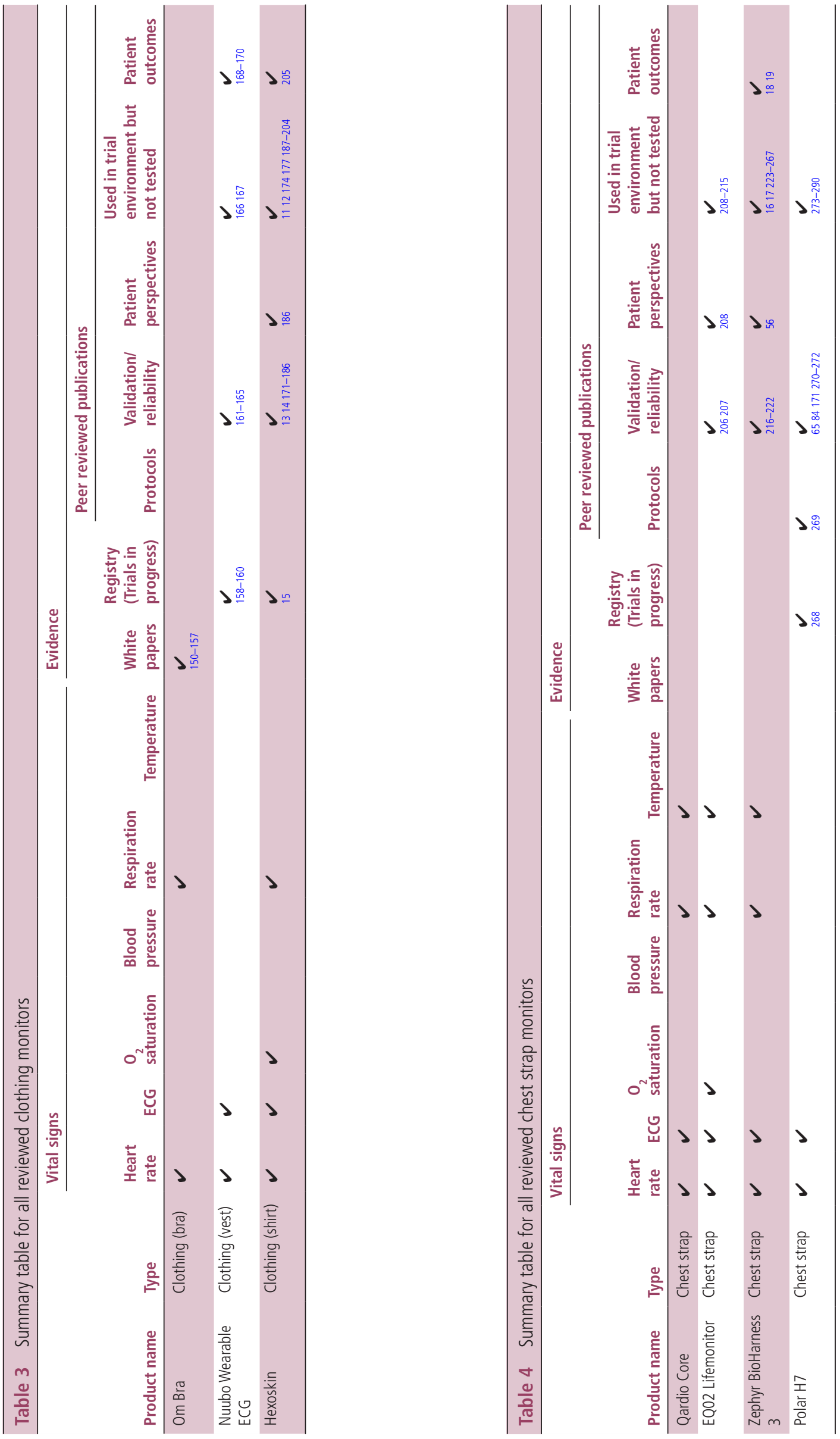

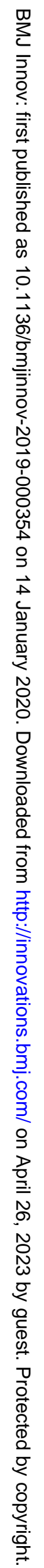




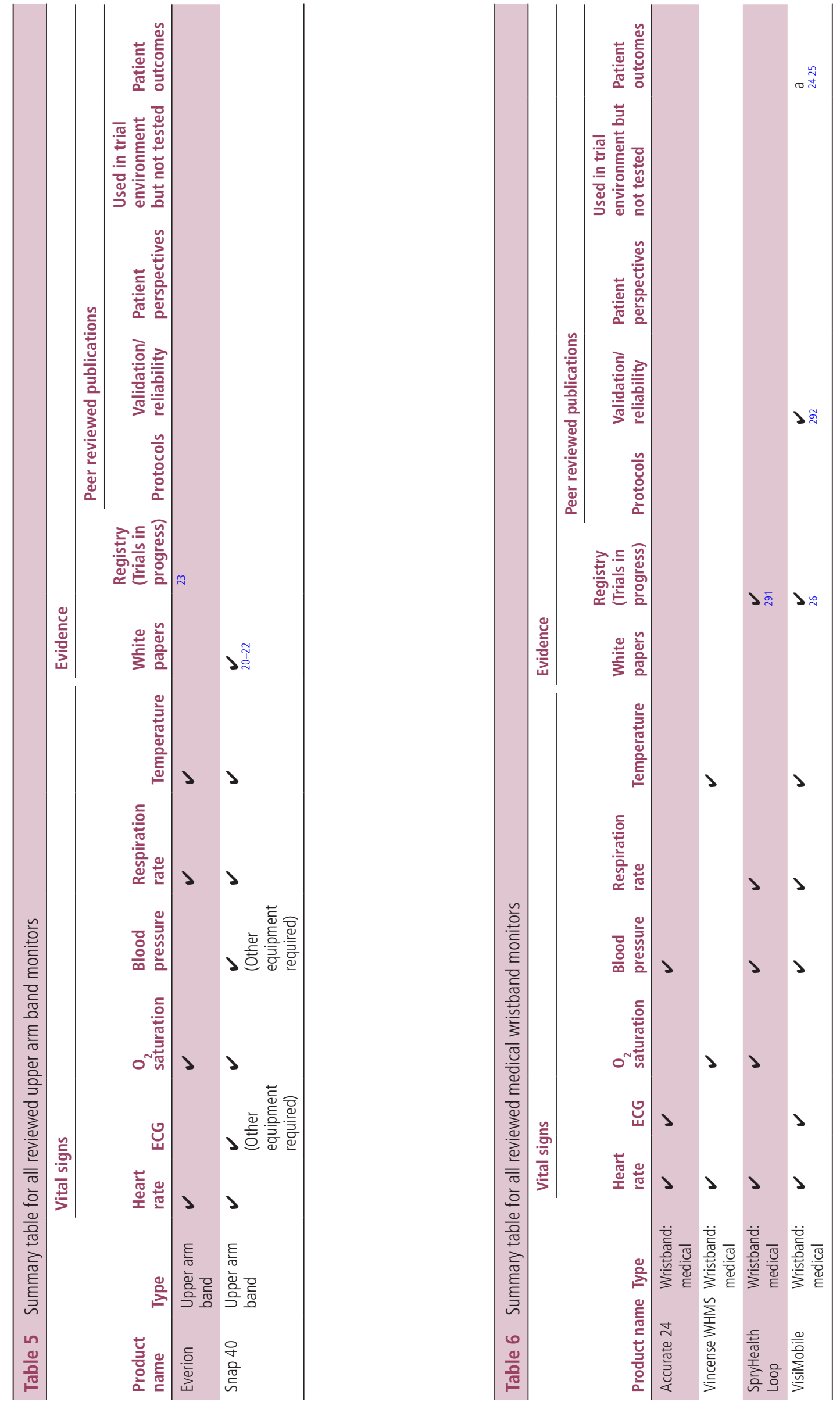

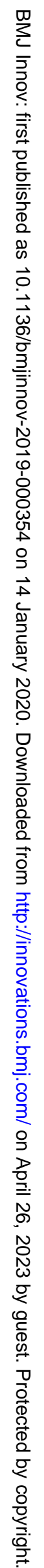




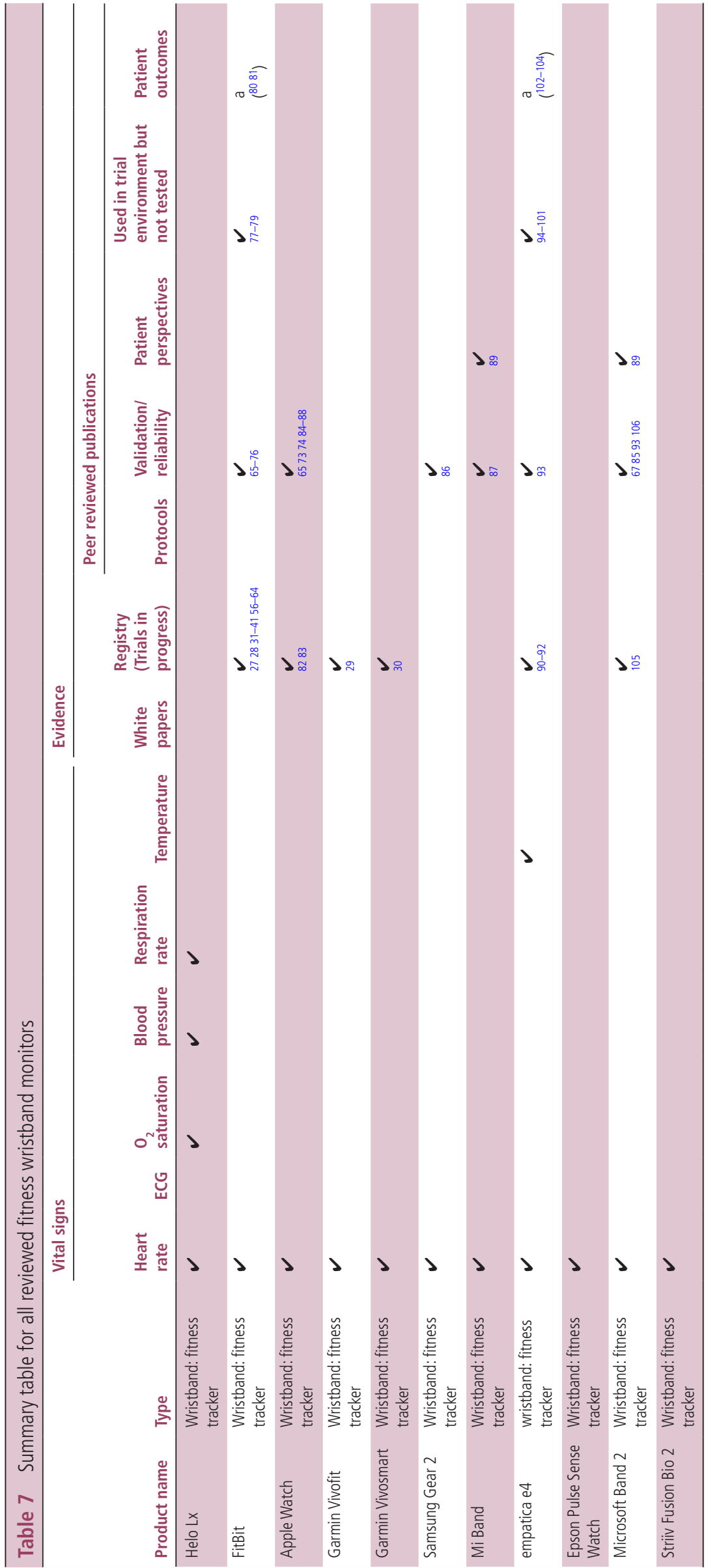

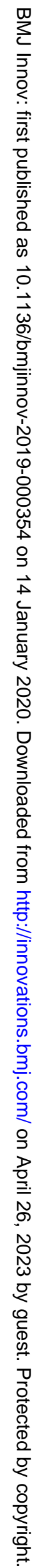


clothing (Nuubo Wearable ECG). Table 3 summarises the vital signs recorded by and the current evidence available for each device.

The clothing monitor with the most evidence was Hexoskin with 42 relevant peer-reviewed publications. Hexoskin's smart shirts are embedded with sensors and have a pocket for a Bluetooth recording device that enables data transmission to the wearer's smartphone. The most common form of peer-reviewed publication for Hexoskin was a trial in which the monitor was used to measure vital signs, but was not evaluated in itself (22 studies). Of the vital sign monitoring functions of Hexoskin, heart rate monitoring was the most common used in these studies and oxygen saturation was the least common. The studies were wide ranging in population and setting, from badminton performance analysis ${ }^{11}$ to the physiology of a paraglider. ${ }^{12}$ The accuracy of Hexoskin was validated in the results of 18 studies using comparative apparatus including pneumotachographs ${ }^{13}$ and Metamax $3 \mathrm{~B}^{14}$ for stationary and moving respiration rate. From these, it can be concluded that the vital signs monitoring functions of Hexoskin are accurate for use in the outpatient setting. These findings may be corroborated by current ongoing trials evaluating the use of Hexoskin in home-based cardiac patients. ${ }^{15}$

The clothing monitor with the least evidence was the OmSignal OmBra with zero relevant peer-reviewed publications and grey literature consisting of eight white papers produced by OmSignal. Of these, all involved validating OmSignal algorithms for analysing the two vital sign monitoring functions of OmBra, heart rate and respiratory rate.

\section{Chest strap monitors}

All four chest straps examined in this study involve devices that strap around the patient's chest with embedded sensors to monitor vital signs. Table 4 summarises the vital signs recorded by and the current evidence available for each device.

Of the chest straps, Zephyr BioHarness 3 had the most relevant peer-reviewed publications. Transmitting data via Bluetooth, this chest strap uses sensors to detect respiratory rate, ECG, heart rate and temperature. Of 57 peer-reviewed publications, 47 described trials where the monitor was used but not tested. In these studies, the Bioharness 3 was used to visualise the physiology of various physical activities from basketball ${ }^{16}$ to cricket. ${ }^{17}$ The results of these studies suggest the Bioharness 3 can be used in ambulatory outpatient monitoring. Two studies found the Bioharness 3 to be equally ${ }^{18}$ or more sensitive $^{19}$ than current hospital methods of respiratory rate detection.

The chest strap with the least evidence was QardioCore with zero literature due to its very recent product release in 2018.

\section{Upper arm band monitors}

Both upper armbands examined in this study strap around the patient's upper arm and consist of cloth straps with a monitoring screen attached. Table 5 summarises the vital signs recorded by and the current evidence available for each device. Snap40 measures blood pressure; this is the least common vital sign measured by the monitors reviewed in this study. The other monitors in this review to measure blood pressure are Accurate 24, SpryHealth Loop, VisiMobile (see table 6) and Helo Lx (see table 7).

Both upper armbands had zero peer-reviewed publications. One white paper assessed the validity and reliability of Snap40 in comparison to a gold standard vital sign monitor as participants went about their daily lives for a week. ${ }^{20}$ The study found that Snap40's measurements correlated with that of the gold standard monitor, demonstrating its ability to be used in real-world applications. Two further studies were conducted in hospital emergency departments. ${ }^{21} 22$ The first of these found that the use of Snap40 reduced time spent on vital sign collection by nurses from an estimated 12.33 hours to 1.2 hours per day. ${ }^{21}$ The study found positive links between less nursing time spent on vital sign collection and patient outcomes. The second study assessed 251 patients in a major UK emergency room for their patient perspective on the device. ${ }^{22}$ This included feedback on their level of comfort wearing the device and their confidence in its monitoring. The Snap40 white papers were funded by Snap40 Ltd.

Everion currently has no evidence in the public domain, but there is a single trial in progress. Conducted by a German university hospital, this is an outpatient validity and reliability study comparing the Everion's ability to detect episodes of atrial fibrillation with that of the gold standard Holter monitor. ${ }^{23}$

\section{Wristband: medical}

Wristbands are by far the most common type of device currently available on the market. Attaching around the patient's wrist, the wristbands studied have been divided into medical and fitness wristbands according to the marketing strategies of their respective companies. Medical wristbands have references to patients and how the devices contribute to health management on their websites. Table 6 summarises the vital signs recorded by and the current evidence available for each medical wristband.

The only medical wristband with relevant peerreviewed publications was VisiMobile with one paper related to validity and reliability and two papers related to patient outcomes, although these were both conducted in the inpatient setting. This was also the wristband measuring the most vital signs: heart rate, ECG, blood pressure, respiratory rate and temperature. This wristband connects to sensors on the patient on body parts such as the thumb and chest. The first of 
the two patient outcome studies was conducted over a 3 -month period in a wing of John Hopkins Hospital, USA, where $40 \%$ of sudden deaths were occurring. ${ }^{24}$ The study found that the introduction of continuous monitoring via Visimobile was associated with a reduction in failure-to-rescue events. The second of these studies surveyed the opinions of nurses on Visimobile after its use on their ward for four consecutive weeks. ${ }^{25}$ In comparison to products such as Hexoskin and Zephyr's Bioharness, Visimobile is unique in that all its clinical trials involve the evaluation of the monitor in a clinical context. This is fitting given its marketing specifically as a wristband for medical use. There is currently one ongoing trial examining the feasibility of its use in a general ward. ${ }^{26}$

Much like upper armbands, medical wristbands are a category very much in development. SpryHealth's Loop was only recently deployed in 2018. Accurate24 is due for release in the summer of 2019. As a result, all three of the other medical wristbands examined have no evidence in the public domain.

\section{Wristband: fitness}

Table 7 summarises the vital signs recorded by and the current evidence available for each device. The fitness wristband with the most evidence was the FitBit with 18 relevant peer-reviewed publications. Of these, 12 studies were related to validity and reliability with a significant proportion of these concerned with the accuracy of the FitBit's heart rate monitoring functions during various forms of exercise. The results of these studies suggest the FitBit provides accurate and reliable heart rate readings that can be used in the ambulatory outpatient setting. Current ongoing trials demonstrate the potential to expand the technology to the healthcare sector. These include a study where the monitor is incorporated into a mobile intervention for pulmonary arterial hypertension, ${ }^{27}$ and a study of patients with mood disorders using the heart rate function of the FitBit Charge series. ${ }^{28}$

The fitness wristbands with the least evidence were Helo Lx, Striiv Fusion Bio 2, Epson Pulse Sense and the Garmin Vivo Series. These had zero relevant peerreviewed publications. Many papers were excluded as they failed to meet the inclusion criteria by focusing on the accelerometer and pedometer functions of these devices. Of these devices, only the Garmin Vivo Series devices had trials in progress, examining the patient perspective of the Garmin Vivo Series devices by measuring adherence and wear time. ${ }^{29} 30$

\section{DISCUSSION}

This review aimed to quantify the evidence available for in-use and upcoming devices for wearable outpatient vital signs monitoring. By identifying the current gaps in the literature and the monitoring functions of these devices, this paper aims to assist clinicians and researchers to select appropriate remote monitoring devices based on existing evidence.

It is apparent that the monitoring of vital signs in the outpatient setting is a developing field, with evidence per monitor ranging from many relevant peer-reviewed publications to zero evidence in the public domain. The FitBit had the most trials in progress, expanding its influence in the field. Gaps in the literature exist with no FitBit patient perspective papers quantifying feasibility and adherence; however, 11 of the $22^{31-41}$ trials in progress will address this with their published results. These will report on compliance, feasibility and adherence from a variety of study populations. ${ }^{31-34}$

Gaps in the literature also exist for upper armbands and medical wristbands. These two types of devices had the fewest relevant peer-reviewed publications. Further research into the use of these wearable outpatient vital signs monitors should look at primarily evaluating their validity and reliability, patient perspectives and patient outcomes when using these devices, in order to validate the products for use in a clinical environment. Gaps in wearable monitor function exist for blood pressure monitors with only five monitors measuring blood pressure. This was followed by $\mathrm{O}_{2}$ saturations with only seven monitors assessing this.

Although there are a multitude of wearable monitors available to the consumer market, there is little evidence of commitment to the routine use of such devices in the healthcare setting. The award of a CE (Conformité Européenne) mark indicates conformity with health, safety and environmental protection standards for products sold within the European Economic Area, but does not guarantee the effectiveness of the product. The level of evidence required to support an application to a national decision-making body such as the National Institute for Health and Care Excellence (NICE) in the UK usually includes clinical trials and health economic analyses. These studies necessitate the commitment of funds, resources and time, thus impacting on the short-term profit margin of small companies. One potential solution to this problem is the partnering of commercial companies with academic institutions to provide high-quality, impactful evidence.

\section{Strengths and limitations}

The main strength of this review is that it is one of the first to give an overview of existing literature on wearable outpatient monitoring devices. Previous research into the field of wearable monitors can be classified into four broad categories: reviews of cardiac functions of monitors; ${ }^{42-45}$ reviews of the activity tracking function such as pedometers and accelerometers of monitors, particularly pertaining to wristband fitness trackers; ${ }^{46-49}$ reviews of respiratory monitoring functions; ${ }^{50}$ and miscellaneous reviews such as those evaluating the use of wearable monitors for monitoring stress, ${ }^{51}$ sleep ${ }^{52}$ and their use in clinical trials. ${ }^{53}$ None 
of these reviews of wearable devices were exclusive to general outpatient monitoring and the monitoring of vital signs. Thus, this review is one of the first of its kind to review wearable outpatient vital signs monitors.

Another strength of this review lies in its scoping nature. Given the developing field, a scoping review allows for quantification of all the evidence relevant to the research question available for each device. The inclusion of a broad range of literature ranging from grey literature such as white papers to registries of trials in progress to peer-reviewed papers allows for a more holistic picture of the evidence. This enables identification of the gaps in the evidence and the product market for wearable outpatient vital signs monitors.

The limitations of this review are the inclusion of only English language publications. Two publications in foreign languages were found, ${ }^{54} 55$ but they were not included due to the language barrier they posed. Furthermore, the quality of each study was not evaluated due to the huge variation in the types of evidence, the study methodologies and the outcomes assessed. An assessment of risk of bias was inappropriate given the scoping nature of the review and the preponderance of grey literature in the search results.

\section{Future research}

Future research should look at aggregating the results of validity and reliability and patient outcome studies for each monitor and between different devices. This would provide a more holistic overview of the potential for clinical use of each device as an outpatient vital signs monitor.

\section{Twitter Candice Downey @MissCDowney}

Contributors CD and SS were involved in the conception of the work and designed the study. SS undertook the data collection and performed the analysis and interpretation. CD, HS and SS drafted the article. All authors (SS, CD, HS and DGJ) were involved in critical revision of the article and have given final approval of the version to be submitted.

Funding $\mathrm{CD}$ is in possession of a Doctoral Research Fellowship (DRF-2016-09-037) supported by the National Institute for Health Research. DGJ is funded by Bowel Cancer UK and RCS England. The research is supported by the NIHR infrastructure at Leeds.

Disclaimer The views expressed in this publication are those of the authors and not necessarily those of the NHS, the National Institute for Health Research, Health Education England or the Department of Health.

Competing interests None declared.

Patient consent for publication Not required.

Provenance and peer review Not commissioned; externally peer reviewed.

Data availability statement Data are available on reasonable request.

ORCID iD

Candice Downey http://orcid.org/0000-0001-9818-8002

\section{REFERENCES}

1 Kause J, Smith G, Prytherch D, et al. A comparison of antecedents to cardiac arrests, deaths and emergency intensive care admissions in Australia and New Zealand, and the United Kingdom--the ACADEMIA study. Resuscitation 2004;62:275-82.

2 Kumar A, Roberts D, Wood KE, et al. Duration of hypotension before initiation of effective antimicrobial therapy is the critical determinant of survival in human septic shock. Crit Care Med 2006;34:1589-96.

3 Odell M. Are early warning scores the only way to rapidly detect and manage deterioration? Nurs Times 2010;106:246.

4 Association AH. American heart association; 2018: 2.

5 Bieganowska K, Kaszuba A, Bieganowski M, et al. PocketECG: a new noninvasive method for continuous and real-time ECG Monitoring-Initial results in children and adolescents. Pediatr Cardiol 2017;38:448-55.

6 Park MHK, de Asmundis C, Chierchia GB, et al. First experience of monitoring with cardiac event recorder electrocardiography Omron system in childhood population for sporadic, potentially arrhythmia-related symptoms. Europace 2011;13:1335-9.

7 Zimlichman E, Szyper-Kravitz M, Shinar Z, et al. Early recognition of acutely deteriorating patients in nonintensive care units: assessment of an innovative monitoring technology. J Hosp Med 2012;7:628-33.

8 Banks J, McArthur J, Gordon G. Flexible monitoring in the management of patient care processes: a pilot study. Lippincotts Case Manag 2000;5:94-103.

9 Gluck S, Chapple L-AS, Chapman MJ, et al. A scoping review of use of wearable devices to evaluate outcomes in survivors of critical illness. Crit Care Resusc 2017;19:197204.

10 Sensium notifications corroborated by manual observations; 2016: 150 .

11 Yong TH, DYW T. Preliminary investigation of movementheart rate relationship using kinect-based badminton performance analysis. In: 2017 International Conference on robotics, automation and sciences (ICORAS). IEEE, 2017: $1-5$.

12 Wilkes M, MacInnis MJ, Hawkes LA, et al. The physiology of Paragliding flight at moderate and extreme altitudes. High Alt Med Biol 2018;19:42-51.

13 Abdallah S, Wilkinson-Maitland C, Waskiw-Ford M. Late Breaking Abstract - Validation of Hexoskin biometric technology to monitor ventilatory responses at rest and during exercise in COPD. ERS International Congress 2017 abstracts, 2017.

14 Elliot CA, Hamlin MJ, Lizamore CA. Validity and reliability of the Hexoskin wearable biometric vest during maximal aerobic power testing in elite cyclists. J Strength Cond Res 2019;33:1437-44.

15 ClinicalTrials.gov. Novel vitality indices derived from the Hexoskin in patients affected with angina undergoing coronary revascularization or medical therapy (NOVA-SKIN); 2015.

16 Kong Z, Qi F, Shi Q. The influence of basketball dribbling on repeated high-intensity intermittent runs. J Exerc Sci Fit 2015;13:117-22.

17 Johnstone JA, Hughes G, Mitchell AC, et al. Accelerometery and heart rate responses of professional Fast-Medium bowlers in one-day and Multi-Day cricket. J Sports Sci Med 2017;16:311-317.

18 Yoon JH, Shah RS, Arnoudse NM, et al. Remote physiological monitoring of acute cocaine exposure. J Med Eng Technol 2014;38:244-50. 
19 Bianchi W, Dugas AF, Hsieh Y-H, et al. Revitalizing a vital sign: improving detection of tachypnea at primary triage. Ann Emerg Med 2013;61:37-43.

20 Rate R, Saturation O, Rate P. Assessment of accuracy in realworld clinical use; 2018: 1-9.

21 Costello J. Free Nursing Time with Automated Vital Sign Monitoring \& Alert Escalation; 2018: 1-5.

22 Mccann C. An evaluation of patient experience of snap40; 2018: 1-6.

23 biovotion. Atrial fibrillation (AFib) study with Biovotion. Available: https://www.biovotion.com/atrial-fibrillation-afibstudy-with-biovotion/

24 Miller PJ. Continuous Monitoring of Patient Vital Signs to Reduce "Failure-to-Rescue" Events Biomedical Instrumentation \& Technology; 2017.

25 Watkins T, Whisman L, Booker P. Nursing assessment of continuous vital sign surveillance to improve patient safety on the medical/surgical unit. J Clin Nurs 2016;25:278-81.

26 ClinicalTrials.gov. Continuous monitoring on the general ward; 2016.

27 ClinicalTrials.gov. A mobile health intervention in pulmonary arterial hypertension (mHealth); 2017.

28 ClinicalTrials.gov. Design and methods of the mood disorder cohort research Consortium (MDCRC) study 2017.

29 ClinicalTrials.gov. Mobile health device study for myeloma patients; 2016.

30 ClinicalTrials.gov. Reducing sedentary behaviour with technology; 2016.

31 ClinicalTrials.gov. Physical activity in children at risk of postthrombotic syndrome (PACT); 2017.

32 ClinicalTrials.gov. Walking in Pregnancy (WiP) - a Social Networking Physical Activity Intervention for Pregnant Obese Women; 2017.

33 ClinicalTrials.gov. Verizon mHealth solution for patients with peripheral artery disease (pad); 2015.

34 ClinicalTrials.gov. Virtual weight loss program for AfricanAmerican breast cancer survivors: preliminary results; 2016.

35 ClinicalTrials.gov. Long-Term usage of the Fitbit ${ }^{\circledR}$ among medical residents; 2016.

36 Increase tolerance for exercise and raise activity through connectedness trial ClinicalTrials.gov; 2017.

37 ClinicalTrials.gov. Tracking physical activity throughout chemotherapy for breast cancer; 2016.

38 ClinicalTrials.gov. Use of a mobile health sensor in an open label trial of lorcaserin for the treatment of cannabis use disorder; 2016.

39 ClinicalTrials.gov. Evaluating the use of wearable biosensors and pros to assess performance status in patients with cancer; 2016.

40 ClinicalTrials.gov. Remote activity monitored by Fitbit charge 2 in investigating daily step and sleep data in participants with head and neck cancer undergoing radiation therapy; 2018.

41 ClinicalTrials.gov. Use of the smart COPD physical activity APP in pulmonary rehabilitation; 2016.

42 Manuscript A, Nanobiomaterials B. Nih public access; 2013: 573-81.

43 Fung E, JÃ€rvelin M-R, Doshi RN, et al. Electrocardiographic patch devices and contemporary wireless cardiac monitoring. Front Physiol 2015;6:1-10.

44 Lobodzinski SS, Laks MM. New devices for very long-term ECG monitoring. Cardiol J 2012;19:210-4.
45 Kelli HM, Witbrodt B, Shah A. The future of mobile health applications and devices in cardiovascular health. Euro Med J Innov 2017;2017:92-7.

46 Tedesco S, Barton J, O'Flynn B, O'Flynn B. A review of activity Trackers for senior citizens: research perspectives, commercial landscape and the role of the insurance industry. Sensors 2017;17. doi:10.3390/s17061277. [Epub ahead of print: 03 Jun 2017].

47 Düking P, Hotho A, Holmberg HC, et al. Comparison of non-invasive individual monitoring of the training and health of athletes with commercially available wearable technologies. Front Physiol 2016;7.

48 Bunn JA, Navalta JW, Fountaine CJ, et al. Current state of commercial wearable technology in physical activity monitoring 2015-2017. Int J Exerc Sci 2018;11:503-15.

49 Henriksen A, Haugen Mikalsen M, Woldaregay AZ, et al. Using fitness Trackers and Smartwatches to measure physical activity in research: analysis of consumer Wrist-Worn Wearables. J Med Internet Res 2018;20:e110.

50 Aliverti A. Wearable technology: role in respiratory health and disease. Breathe 2017;13:e27-36.

51 Seoane F, Mohino-Herranz I, Ferreira J, et al. Wearable biomedical measurement systems for assessment of mental stress of combatants in real time. Sensors 2014;14:7120-41.

52 Peake JM, Kerr G, Sullivan JP. A critical review of consumer wearables, mobile applications, and equipment for providing biofeedback, monitoring stress, and sleep in physically active populations. Front Physiol 2018;9:1-19.

53 Izmailova ES, Wagner JA, Perakslis ED. Wearable devices in clinical trials: hype and hypothesis. Clin Pharmacol Ther 2018;104:42-52.

54 Mont-Royal -Montréal C. Présentations par affiche Recueil d'abrégés-Présentations par affiche; 2017.

55 Wirkung von Herzkohärenztraining auf; 2016.

56 Brannon EE, Cushing CC, Crick CJ, et al. The promise of wearable sensors and ecological momentary assessment measures for dynamical systems modeling in adolescents: a feasibility and acceptability study. Transl Behav Med 2016;6:558-65.

57 ClinicalTrials.gov. Wearable sensor for biometrics during locoregional therapy for head and neck cancer; 2018.

58 ClinicalTrials.gov. Role of Centrum ${ }^{\circledR}$ Silver ${ }^{\circledR}$ in improving micronutrient status in older men; 2016.

59 ClinicalTrials.gov. Behavioral weight and symptom management for breast cancer survivors and partners; 2013.

60 ClinicalTrials.gov. Post-Market surveillance with a novel mHealth platform; 2018.

61 ClinicalTrials.gov. Fitbit and Social Support in Knee Replacement Patients \& Buddies; 2017.

62 Good Clinical Practice. Increasing exercise adherence after percutaneous coronary intervention with the Fitbit charge HR device (incentive); 2017.

63 ClinicalTrials.gov. Evaluation of outcomes of crt and MitraClip for treatment of low ejection fraction and functional mitral regurgitation in HF; 2016.

64 ClinicalTrials.gov. Assessing physical fitness in cancer patients with cardiopulmonary exercise testing and wearable data generation; 2016.

65 Bai Y, Hibbing P, Mantis C, et al. Comparative evaluation of heart rate-based monitors: apple Watch vs Fitbit charge HR. $J$ Sports Sci 2018;36:1734-41.

66 Lee H-A, Lee H-J, Moon J-H, et al. Comparison of wearable activity Tracker with actigraphy for sleep evaluation and 
circadian Rest-Activity rhythm measurement in healthy young adults. Psychiatry Investig 2017;14:179-85.

67 Stahl SE, An H-S, Dinkel DM, et al. How accurate are the wrist-based heart rate monitors during walking and running activities? are they accurate enough? BMJ Open Sport Exerc Med 2016;2:e000106.

68 Pelizzo G, Guddo A, Puglisi A, et al. Accuracy of a WristWorn heart rate sensing device during elective pediatric surgical procedures. Children;5:38.

69 Kroll RR, Boyd JG, Maslove DM. Accuracy of a WristWorn wearable device for monitoring heart rates in hospital inpatients: a prospective observational study. J Med Internet Res 2016;18:e253.

70 Jo E, Lewis K, Directo D, et al. Validation of biofeedback Wearables for Photoplethysmographic heart rate tracking. J Sports Sci Med 2016;15:540-547.

71 Leth S, Hansen J, Nielsen O, et al. Evaluation of commercial self-monitoring devices for clinical purposes: results from the future patient trial, phase I. Sensors; 17:211.

72 Gorny AW, Liew SJ, Tan CS, et al. Fitbit charge HR wireless heart rate monitor: validation study conducted under freeliving conditions. JMIR Mhealth Uhealth 2017;5:e157.

73 Dooley EE, Golaszewski NM, Bartholomew JB. Estimating accuracy at exercise intensities: a comparative study of selfmonitoring heart rate and physical activity wearable devices. JMIR Mhealth Uhealth 2017;5:e34.

74 Wallen MP, Gomersall SR, Keating SE, et al. Accuracy of heart rate watches: implications for weight management. PLoS One 2016;11:e0154420.

75 Cadmus-Bertram L, Gangnon R, Wirkus EJ, et al. The accuracy of heart rate monitoring by some Wrist-Worn activity Trackers. Ann Intern Med 2017;166:610.

76 Benedetto S, Caldato C, Bazzan E, et al. Assessment of the Fitbit charge 2 for monitoring heart rate. PLoS One 2018;13:e0192691.

77 De Zambotti M, Baker FC, Willoughby AR, et al. Measures of sleep and cardiac functioning during sleep using a multi-sensory Commercially-Available Wristband in Adolescents Wearable technology to measure sleep and cardiac functioning. Physiol Behav 2016;158:143-9.

78 Sprint G, Cook D, Weeks D, et al. Analyzing Sensor-Based time series data to track changes in physical activity during inpatient rehabilitation. Sensors 2017;17:2219.

79 ClinicalTrials.gov. Objective assessment of physical activity during chemotherapy for breast cancer; 2017.

80 Lee J-E, Lee DH, Oh TJ, et al. Clinical feasibility of continuously monitored data for heart rate, physical activity, and sleeping by wearable activity Trackers in patients with thyrotoxicosis: protocol for a prospective longitudinal observational study. JMIR Res Protoc 2018;7:e49.

81 Kroll RR, McKenzie ED, Boyd JG, et al. Use of wearable devices for post-discharge monitoring of ICU patients: a feasibility study. J Intensive Care 2017;5:64.

82 ClinicalTrials.gov. Apple heart study: assessment of Wristwatch-Based Photoplethysmography to identify cardiac arrhythmias; 2017.

83 Testing the effects of movement based interventions on brainbody mechanisms in Fatigued cancer survivors; 2017.

84 GILLINOV S, ETIWY M, WANG R, et al. Variable accuracy of wearable heart rate monitors during aerobic exercise. Med Sci Sport Exerc 2017;49:1697-703.
85 Shcherbina A, Mattsson C, Waggott D, et al. Accuracy in Wrist-Worn, Sensor-Based measurements of heart rate and energy expenditure in a diverse cohort. J Pers Med 2017;7:3.

86 El-Amrawy F, Nounou MI. Are currently available wearable devices for activity tracking and heart rate monitoring accurate, precise, and medically beneficial? Healthc Inform Res 2015;21:315-20.

87 Xie J, Wen D, Liang L, et al. Evaluating the validity of current mainstream wearable devices in fitness tracking under various physical activities: comparative study. JMIR Mhealth Uhealth 2018;6:e94.

88 Wang R, Blackburn G, Desai M, et al. Accuracy of WristWorn heart rate monitors. JAMA Cardiol 2017;2:104.

89 Puri A, Kim B, Nguyen O, et al. User acceptance of Wrist-Worn activity Trackers among community-dwelling older adults: mixed method study. JMIR Mhealth Uhealth 2017;5:e173.

90 ClinicalTrials.gov. Opioid physiology project; 2018.

91 ClinicalTrials.gov. Probiotics and oxytocin nasal spray on social behaviors of autism spectrum disorder (ASD) children; 2017.

92 ClinicalTrials.gov. Treatment of mitochondrial dysfunction in Rett syndrome with Triheptanoin; 2016.

93 Kos M, Li X, Khaghani-Far I. Can accelerometry data improve estimates of heart rate variability from wrist pulse PPG sensors?*. Conf Proc IEEE Eng Med Biol Soc, 2017.

94 Brotherhood E, Ball P, Camic PM, et al. Preparatory planning framework for created out of mind: shaping perceptions of dementia through art and science. Wellcome Open Res 2017;2:108.

95 Krämer N, Sobieraj S, Feng D, et al. Being Bullied in virtual environments: experiences and reactions of male and female students to a male or female Oppressor. Front Psychol 2018;9:253.

96 Ali M, Machot F, Mosa A, et al. A globally generalized emotion recognition system involving different physiological signals. Sensors 2018;18:1905.

97 Salamone F, Belussi L, Currò C, et al. Integrated Method for Personal Thermal Comfort Assessment and Optimization through Users' Feedback, IoT and Machine Learning: A Case Study ${ }^{\dagger}$. Sensors 2018;18. doi:10.3390/s18051602. [Epub ahead of print: 17 May 2018].

98 Hao T, Walter KN, Ball MJ, et al. StressHacker: towards practical stress monitoring in the wild with Smartwatches. AMIA Annu Symp Proc 2017;2017:830-838.

99 Vicary S, Sperling M, von Zimmermann J, et al. Joint action aesthetics. PLoS One 2017;12:e0180101.

100 Sim S, Koh M, Joo K, et al. Estimation of thermal sensation based on wrist skin temperatures. Sensors 2016;16:420.

101 Chrisinger BW, King AC. Stress experiences in neighborhood and social environments (sense): a pilot study to integrate the quantified self with citizen science to improve the built environment and health. Int J Health Geogr 2018;17:17.

102 Corino VDA, Laureanti R, Ferranti L, et al. Detection of atrial fibrillation episodes using a wristband device. Physiol Meas 2017;38:787-99.

103 Chintha KK, Indic P, Chapman B, et al. Wearable biosensors to evaluate recurrent opioid toxicity after naloxone administration: a Hilbert transform approach. Proc Annu Hawaii Int Conf Syst Sci 2018;2018:3247-52.

104 Siirtola P, Koskimäki H, Mönttinen H, et al. Using sleep time data from wearable sensors for early detection of migraine attacks. Sensors 2018;18:1374. 
105 Arousal response tool for neurological disease.

106 Thiebaud RS, Funk MD, Patton JC, et al. Digital health brief communication validity of wrist-worn consumer products to measure heart rate and energy expenditure.

107 Selvaraj N. Psychological acute stress measurement using a wireless adhesive biosensor. In: 2015 37th annual International Conference of the IEEE engineering in medicine and biology Society (EmbC. IEEE, 2015: 3137-40.

108 Selvaraj N, Doan T. Performance of energy expenditure assessment using a chest-worn wireless patch sensor IEEE; 2015: 238-41.

109 Selvaraj N, Narasimhan R. Automated prediction of the apnea-hypopnea index using a wireless patch sensor. Conf Proc IEEE Eng Med Biol Soc 2014;2014:1897-900.

110 Chan AM, Selvaraj N, Ferdosi N, et al. Wireless patch sensor for remote monitoring of heart rate, respiration, activity, and falls. Conf Proc IEEE Eng Med Biol Soc 2013;2013:6115-8.

111 Chan AM, Ferdosi N, Narasimhan R. Ambulatory respiratory rate detection using ECG and a triaxial accelerometer. Conf Proc IEEE Eng Med Biol Soc 2013;2013:4058-61.

112 Selvaraj N. Long-Term remote monitoring of vital Signsusing a wireless patch sensor. 2014 Health Innovations and Pointof-Care Technologies Conference, Seattle, Washington USA, 2014:8-10.

113 ClinicalTrials.gov. Evaluation of the BodyGuardian end-toend remote monitoring platform in an outpatient heart failure population; 2013.

114 ClinicalTrials.gov. Assessment of the BodyGuardian remote monitoring platform in elderly healthy subjects; 2014.

115 ClinicalTrials.gov. Potassium and cardiac rhythm trends in maintenance hemodialysis: a multicenter, prospective, observational study; 2015.

116 ClinicalTrials.gov. Continuous data collection and analysis for stroke prevention using a wearable sensor; 2018.

117 ClinicalTrials.gov. BodyGuardian respiration and activity validation testing; 2014.

118 ClinicalTrials.gov. A study to evaluate the effect of the transient receptor potential vanilloid 4 (TRPV4) channel blocker, GSK2798745, on pulmonary gas transfer and respiration in patients with congestive heart failure; 2018.

119 Bruce CJ, Ladewig DJ, Somers VK, et al. Remote electrocardiograph monitoring using a novel adhesive strip sensor: a pilot study. World J Cardiol 2016;8:559.

120 Sensium Healthcare. SensiumVitals ${ }^{\circledR}$ respiration rate algorithm; 2016: 4-7.

121 Sensium Healthcare. SensiumVitals ${ }^{\circledR}$ heart rate algorithm; 2018: 3-6.

122 Defined P. Communication protocol; 2018: 1-4.

123 John SSaint John' S health center pilot study; 2014: 1-8.

124 Backed I, Evidence BY. Sensium Whitepaper - The Deteriorating Patient; 2018: 1-5.

125 Hernandez-Silveira M, Ahmed K, Ang S-S, et al. Assessment of the feasibility of an ultra-low power, wireless digital patch for the continuous ambulatory monitoring of vital signs. BMJ Open 2015;5:e006606.

126 Hernandez-Silveira M, Wieczorkowski-Rettinger K, Ang S, et al. Preliminary assessment of the SensiumVitals ${ }^{\circledR}$ : a lowcost wireless solution for patient surveillance in the general wards. Conf Proc IEEE Eng Med Biol Soc 2015;2015:4931-7.

127 Downey CL, Brown JM, Jayne DG, et al. Patient attitudes towards remote continuous vital signs monitoring on general surgery wards: an interview study. Int J Med Inform 2018;114:52-6.
128 Heart CN, Caregivers T, Early N. Sensium: selected case studies; 2012.

129 Sensium. Sensium notifications lead to meningitis diagnosis; 2016.

130 Sensium. Sensium detects tachycardia episode between observation rounds; 2016.

131 Sensium. Sensium notifications lead to atrial fibrillation diagnosis; 2016.

132 Sensium. Sensium detects opioid induced respiratory complication; 2016.

133 Sensium. Sensium detects sepsis; 2016.

134 Sensium. Sensium notifications lead to pulmonary oedema diagnosis; 2016.

135 Sensium. Sensium detects opioid induced respiratory depression; 2016: 65.

136 Care S. Optimising the National Health Service Through Remote Vital Signs Monitoring : zensor January 2013; 2013: $1-11$.

137 Claes J, Buys R, Woods C, et al. Pathway I: design and rationale for the investigation of the feasibility, clinical effectiveness and cost-effectiveness of a technology-enabled cardiac rehabilitation platform. BMJ Open 2017;7:e016781.

138 ClinicalTrials.gov. Impact of an intensive monitoring strategy in symptomatic patients with suspected arrhythmia; 2016.

139 Singh B, Rao H, Pandurangi U, et al. Identifying highrisk patients post myocardial infarction with reduced left ventricular function using external loop recorders (INSPIREELR study). J Am Coll Cardiol 2018;71:A432.

140 ClinicalTrials.gov. Mobile health monitoring solution for heart failure patients; 2015.

141 ClinicalTrials.gov. Recurrent atrial fibrillation; 2018.

142 ClinicalTrials.gov. Extended ambulatory monitoring improves risk stratification in hypertrophic cardiomyopathy; 2017.

143 ClinicalTrials.gov. Detection of atrial fibrillation after cardiac surgery; 2016.

144 ClinicalTrials.gov.. SEEQTM performance study; 2015.

145 Shareghi S, Tavakol M, Lindborg K. Abstract 16078: SEEQ mobile cardiac telemetry associated with a high yield of clinically relevant arrhythmias in patients with suspected arrhythmia. Circulation 2018;134.

146 Engel JM, Chakravarthy BLN, Rothwell D, et al. SEEQ ${ }^{\text {TM }}$ MCT wearable sensor performance correlated to skin irritation and temperature. Conf Proc IEEE Eng Med Biol Soc 2015;2015:2030-3.

147 Engel JM, Chakravarthy N, Katra RP, et al. Estimation of patient compliance in application of adherent mobile cardiac telemetry device. Conf Proc IEEE Eng Med Biol Soc 2011;2011:1536-9.

148 Engel JM, Mehta V, Fogoros R, et al. Study of arrhythmia prevalence in NUVANT Mobile Cardiac Telemetry system patients. In: 2012 annual International Conference of the IEEE engineering in medicine and biology Society. IEEE, 2012: 2440-3.

149 Engel JM, Chakravarthy N, Nosbush G, et al. Comparison of arrhythmia prevalence in NUVANT mobile cardiac telemetry system patients in the US and India. Conf Proc IEEE Eng Med Biol Soc 2014;2014:2730-3.

150 Note O. OMsignal heart rate variability detection; 2018: 6-9.

151 Note O. Validation of breathing rate algorithm during running; 2017.

152 Note O. Validation of breathing depth and ventilation measure during running; 2017.

153 Note O. ECG-Based human identification; 2017: 1-5. 
154 Note O. Automated detection of anaerobic and ventilatory thresholds from biometric data; 2017.

155 Note O. Deep Convolutional neural network for ECG-Based human identification; 2017: 7-10.

156 Note O. Daily stress prediction using heart rate variability metrics; 2017: 3-5.

157 Table I. Heart rate algorithm verification; 2017: 1-3.

158 ClinicalTrials.gov. Atrial fibrillation research in Catalonia; 2017.

159 ClinicalTrials.gov. Randomized evaluation of Redo ablation procedures of atrial fibrillation with focal impulse and rotor modulation guided procedures; 2016.

160 ClinicalTrials.gov. Elr monitoring against permanent pacemaker in atrial fibrillation; 2015.

161 Perez de Isla L, Lennie V, Quezada M, et al. New generation dynamic, wireless and remote cardiac monitorization platform: a feasibility study. Int J Cardiol 2011;153:83-5.

162 Balsam P, Lodziński P, Tymińska A, et al. Study design and rationale for biomedical shirt-based electrocardiography monitoring in relevant clinical situations: ECG-shirt study. Cardiol J 2018;25:52-9.

163 Olmos C, Franco E, Suárez-Barrientos A, et al. Wearable wireless remote monitoring system: an alternative for prolonged electrocardiographic monitoring. Int J Cardiol 2014;172:e43-4.

164 Fernandez S, Haas N, Lehner A, et al. Filling the gap in the detection and management of dysrhythmia: the life vest. Thorac Cardiovasc Surg 2017;65:S111-42.

165 Fabregat-Andres O, Munoz-Macho A, Adell-Beltran G, et al. Evaluation of a new Shirt-Based electrocardiogram device for cardiac screening in soccer players: comparative study with treadmill Ergospirometry. Cardiol Res 2014;5:101-7.

166 Verbiest-van Gurp N, van Bladel PJM, van Kesteren HAM, et al. Current practice of Dutch cardiologists in detecting and diagnosing atrial fibrillation: results of an online case vignette study. Neth Heart J 2017;25:567-73.

167 Ferrero-de-Loma-Osorio Ángel, García-Fernández A, Castillo-Castillo J, et al. Time-to-Effect-Based dosing strategy for Cryoballoon ablation in patients with paroxysmal atrial fibrillation: results of the plusONE multicenter randomized controlled Noninferiority trial. Circ Arrhythm Electrophysiol 2017;10:e05318.

168 Locati ET, Ardito C, Cecchi F, et al. P5504Diagnostic yield of 7-days versus 21-days recordings by using a new wearable wireless continuous ambulatory ECG recorder. Eur Heart J 2017;38.

169 Pagola J, Juega J, Francisco-Pascual J, et al. Yield of atrial fibrillation detection with textile wearable Holter from the acute phase of stroke: pilot study of Crypto-AF registry. Int $J$ Cardiol 2018;251:45-50.

170 Bravo-Escobar R, González-Represas A, Gómez-González $\mathrm{AM}$, et al. Effectiveness and safety of a home-based cardiac rehabilitation programme of mixed surveillance in patients with ischemic heart disease at moderate cardiovascular risk: a randomised, controlled clinical trial. BMC Cardiovasc Disord 2017;17.

171 Al Sayed C, Vinches L, Hallé S. Validation of a Wearable Biometric System's Ability to Monitor Heart Rate in Two Different Climate Conditions under Variable Physical Activities. Ehealth Telecommun Syst Netw 2017;06:19-30.

172 Montes J, Stone TM, Manning JW, et al. Using Hexoskin wearable technology to obtain body metrics during TRAIL hiking. Int J Exerc Sci 2015;8:425-430.
173 Bellone GJ, Plano SA, Cardinali DP, et al. Comparative analysis of actigraphy performance in healthy young subjects. Sleep Science 2016;9:272-9.

174 Beltrame T, Amelard R, Wong A, et al. Prediction of oxygen uptake dynamics by machine learning analysis of wearable sensors during activities of daily living. Sci Rep 2017;7:45738.

175 Villar R, Beltrame T, Hughson RL. Validation of the Hexoskin wearable vest during lying, sitting, standing, and walking activities. Appl Physiol Nutr Metab 2015;40:1019-24.

176 Banerjee T, Peterson M, Oliver Q, et al. Validating a commercial device for continuous activity measurement in the older adult population for dementia management. Smart Health 2018;5-6:51-62.

177 Bougouin W, Marijon E, Planquette B, et al. Proceedings of Réanimation 2017, the French intensive care Society international Congress. Ann Intensive Care 2017;7:1.

178 Fox N, Davis JE, Brown A, et al. Assessing physiological function during a high-altitude HIKE using real-time monitoring. Med Sci Sport Exerc 2017;49:764.

179 Mezghani N, Ouakrim Y, Islam MR, et al. Context aware adaptable approach for fall detection bases on smart textile. In: 2017 IEEE EMBS International Conference on Biomedical \& Health Informatics (BHI. IEEE, 2017: 473-6.

180 Phillips M, Phillips MB, Beach J, et al. Reliability and validity of the Hexoskin wearable body metrics telemetry Shirt. $J$ Sport Hum Perform 2017;11.

181 Pion-Massicotte J, Chicoine M, Chevrier É, et al. 0069 Development and validation of an algorithm for the study of sleep using a biometric shirt in young healthy adults. Sleep 2017;40:A26-7.

182 DYW T, Yong TH. Suitability of smartshirt by Hexoskin to monitor heart rate for racket sports. In: 2017 International Conference on robotics, automation and sciences (ICORAS. IEEE, 2017: 1-4.

183 Beltrame T, Hughson RL. Aerobic system analysis based on oxygen uptake and hip acceleration during random overground walking activities. Am J Physiol Regul Integr Comp Physiol 2017;312:R93-100.

184 Beltrame T, Amelard R, Wong A, et al. Extracting aerobic system dynamics during unsupervised activities of daily living using wearable sensor machine learning models. J Appl Physiol 2018;124:473-81.

185 Banerjee T, Anantharam P, Romine W. Evaluating a potential commercial tool for healthcare application for people with dementia. International Conference on Health Informatics and Medical Systems, At Las Vegas, NV, 2015.

186 Harrison EM, Bessman SC, Markwald RR, et al. Efficacy of a smart textile Shirt: developing a sleep health screening tool for military populations gaps addressed.

187 Barker L, Mercer J. Measuring acceleration of a Trampoline Circus act during training and In-Show using wearable technology. Med Sci Sport Exerc 2017;49:763.

188 Bennett SL, Goubran R, Knoefel F. Adaptive eulerian video magnification methods to extract heart rate from thermal video. In: 2016 IEEE International Symposium on medical measurements and applications (MeMeA). IEEE, 2016: 1-5.

189 Chatty L, Omurtag A, Roy R. Tracking mental workload by multimodal measurements during minimally invasive surgery training Sages; 2017.

190 Gross MJ, Hall R, Bringer JD, et al. Resonant frequency training in elite sport: a case study example. J Sport Psychol Action 2017;8:173-83. 
191 Gutta S, Cheng Q, Benjamin BA. Control mechanism modeling of human cardiovascular-respiratory system. In: 2015 IEEE global conference on signal and information processing (GlobalSIP). IEEE, 2015: 918-22.

192 Hunt MG, Rushton J, Shenberger E, et al. Positive effects of diaphragmatic breathing on physiological stress reactivity in varsity athletes. J Clin Sport Psychol 2018;12:27-38.

193 Le Floch T, Nadeau S, Landau K. Aircraft deicing in open baskets:Study of the effects of activities on heart rate variability; 2018.

194 Slamon NB, Penfil SH, Nadkarni VM, et al. A Prospective Pilot Study of the Biometrics of Critical Care Practitioners during Live Patient Care using a Wearable "Smart Shirt". J Intensive Crit Care 2018;04:10.

195 Rahman T, Adams AT, Ravichandran RV. DoppleSleep: a contactless Unobtrusive sleep sensing system using shortrange Doppler radar. The 2015 ACM International Joint Conference on Pervasive and Ubiquitous Computing, Osaka, Japan, 2015.

196 Salar M, Capanoglu MF, Sherman A, et al. Training related risk factors of firefighters. Proc Hum Factors Ergon Soc Annu Meet 2017;61:1844-7.

197 Schneider BA. Gait analysis from wearable devices using image and signal processing; 2017.

198 Slamon N, Nadkarni V, Penfil S, et al. Analysis of heart rate and heart rate variability during live pediatric intensive care activities lower serum albumin at ICU admission predicts postoperative AKI following 2017;46.

199 Slamon N, Penfil S, Deutsch E, et al. 1131: biometric stress tracking and analysis during acute ICU care using a novel biosensing garment. Critical Care Medicine 2016;44.

200 Smith M, Denning M, Zagrodnik J, et al. A Comparison of Pickleball and Walking: A Pilot Study: 356 Board \#193 June 1, 11: 00 AM - 12: 30 PM. Am J Sports Med 2016;48.

201 Speckhard AD, Knous JL, Coughlin AM. Comparison of physiological demands of Basketball practice sessions to a Preseason game. Med Sci Sport Exerc 2017;49:622.

202 Wilson SL, Bradley R, Shin JM, et al. Heart rate variability in aerobically trained subjects vs. untrained subjects in hypoxic and normoxic conditions. Off J Am Coll Sport Med 2016;48:310.

203 Marjanovic NS, De Simone A, Jegou G, et al. A new global and comprehensive model for ICU ventilator performances evaluation. Ann Intensive Care 2017;7:68.

204 Tobon V. DP, Falk TH, Maier M. MS-QI: a modulation spectrum-based ECG quality index for telehealth applications. IEEE Trans Biomed Eng 2016;63:1613-22.

205 van Leuteren R, Fabius T, de Jongh F. Detecting dynamic hyperinflation in COPD patients using a smart shirt: a pilot study. ERS International Congress 2017, 2017.

206 Akintola AA, van de Pol V, Bimmel D, et al. Comparative analysis of the Equivital EQ02 Lifemonitor with Holter ambulatory ECG device for continuous measurement of ECG, heart rate, and heart rate variability: a validation study for precision and accuracy. Front Physiol 2016;7:391.

207 Liu Y, Zhu SH, Wang GH, et al. Validity and reliability of multiparameter physiological measurements recorded by the Equivital LifeMonitor during activities of various intensities. $J$ Occup Environ Hyg 2013;10:78-85.

208 Sun P, Wergeles NM, Zhang C, et al. ADA - Automatic Detection of Alcohol Usage for Mobile Ambulatory Assessment. In: 2016 IEEE International Conference on smart computing (SMARTCOMP). IEEE, 2016: 1-5.
209 Magnuson V, Wang Y, Schork N. Normalizing sleep quality disturbed by psychiatric polypharmacy: a single patient open trial (spot). F1000Res 2016;5:132.

210 Tang MW, van Nierop FS, Koopman FA, et al. Single vagus nerve stimulation reduces early postprandial C-peptide levels but not other hormones or postprandial metabolism. Clin Rheumatol 2018;37:505-14.

211 Hunt AP, Billing DC, Patterson MJ, et al. Heat strain during military training activities: the dilemma of balancing force protection and operational capability. Temperature 2016;3:307-17.

212 Benedetti F, Dogue S. Different placebos, different mechanisms, different outcomes: lessons for clinical trials. PLoS One 2015;10:e0140967.

213 Jansen SW, Akintola AA, Roelfsema F, et al. Human longevity is characterised by high thyroid stimulating hormone secretion without altered energy metabolism. Sci Rep 2015;5.

214 Heesch MW, Shute RJ, Kreiling JL, et al. Transcriptional control, but not subcellular location, of PGC- $1 \alpha$ is altered following exercise in a hot environment. J Appl Physiol 2016;121:741-9.

215 Sandsund M, Høye EU, Heidelberg CT, et al. Work environment and health in the Norwegian fishing fleet - a field study on board deep-sea fishing vessels. Extrem Physiol Med 2015;4:A48.

216 Rawstorn JC, Gant N, Warren I, et al. Measurement and data transmission validity of a Multi-Biosensor system for real-time remote exercise monitoring among cardiac patients. JMIR Rehabil Assist Technol 2015;2:e2.

217 Johnstone JA, Ford PA, Hughes G, et al. Field based reliability and validity of the bioharness ${ }^{\mathrm{TM}}$ multivariable monitoring device. J Sports Sci Med 2012;11:643-52.

218 Johnstone JA, Ford PA, Hughes G, et al. Bioharness $\left({ }^{\mathrm{TM}}\right)$ multivariable monitoring device: part. I: validity. J Sports Sci Med 2012;11:400-8.

219 Rawstorn JC, Gant N, Meads A, et al. Remotely delivered Exercise-Based cardiac rehabilitation: design and content development of a novel mHealth platform. JMIR Mhealth Uhealth 2016;4:e57.

220 Johnstone JA, Ford PA, Hughes G, et al. Bioharness $\left({ }^{\mathrm{TM}}\right)$ Multivariable Monitoring Device: Part. II: Reliability. J Sports Sci Med 2012;11:409-17.

221 Kim J-H, Roberge R, Powell JB, et al. Measurement accuracy of heart rate and respiratory rate during graded exercise and sustained exercise in the heat using the Zephyr BioHarness. Int J Sports Med 2013;34:497-501.

222 Seo Y, DiLeo T, Powell JB, et al. Comparison of estimated core body temperature measured with the BioHarness and rectal temperature under several heat stress conditions. $J$ Occup Environ Hyg 2016;13:612-20.

223 Imtiaz SA, Mardell J, Saremi-Yarahmadi S, et al. Ecg artefact identification and removal in mHealth systems for continuous patient monitoring. Healthc Technol Lett 2016;3:171-6.

224 De La Garza R, Yoon JH, Thompson-Lake DGY, et al. Treadmill exercise improves fitness and reduces craving and use of cocaine in individuals with concurrent cocaine and tobacco-use disorder. Psychiatry Res 2016;245:133-40.

225 Ortiz-Vigon Uriarte IdeL, Garcia-Zapirain B, Garcia-Chimeno Y. Game design to measure reflexes and attention based on biofeedback multi-sensor interaction. Sensors 2015;15:652048 
226 Turksoy K, Paulino TML, Zaharieva DP, et al. Classification of physical activity: information to artificial pancreas control systems in real time. J Diabetes Sci Technol 2015;9:1200-7.

227 Stenerson M, Cameron F, Wilson DM, et al. The impact of Accelerometer and heart rate data on hypoglycemia mitigation in type 1 diabetes. J Diabetes Sci Technol 2014;8:64-9.

228 Padhi S, Patel N, Driscoll D, et al. Prevalence of cardiac arrhythmias in a community based chiropractic practice. $J$ Can Chiropr Assoc 2014;58:238-45.

229 Simjanoska M, Gjoreski M, Gams M, et al. Non-Invasive blood pressure estimation from ECG using machine learning techniques. Sensors $; 18: 1160$.

230 Nazari G, MacDermid JC, Sinden KE, et al. The relationship between physical fitness and simulated Firefighting task performance. Rehabil Res Pract 2018;2018:1-7.

231 Hettinga FJ, Konings MJ, Cooper CE. Differences in muscle oxygenation, perceived fatigue and recovery between Long-Track and Short-Track speed skating. Front Physiol 2016;7:619.

232 Rodes CE, Chillrud SN, Haskell WL, et al. Predicting adult pulmonary ventilation volume and wearing compliance by On-Board Accelerometry during personal level exposure assessments. Atmos Environ 2012;57:126-137.

233 Williamon A, Aufegger L, Eiholzer H, et al. Simulating and stimulating performance: introducing distributed simulation to enhance musical learning and performance. Front Psychol 2014;5.

234 Rai B, Kaur J. Human factor studies on a Mars analogue during Crew 100b international Lunar exploration Working group EuroMoonMars Crew: proposed new approaches for future human space and Interplanetary missions. $\mathrm{N} \mathrm{Am} \mathrm{J} \mathrm{Med}$ Sci 2012;4:548-57.

235 Taamneh S, Tsiamyrtzis P, Dcosta M, et al. A multimodal dataset for various forms of distracted driving. Sci Data 2017;4:170110.

236 Constantini K, Stickford ASL, Bleich JL, et al. Synchronizing gait with cardiac cycle phase alters heart rate response during running. Med Sci Sport Exerc 2018;50:1046-53.

237 Rigoli LM, Holman D, Spivey MJ, et al. Spectral convergence in tapping and physiological fluctuations: coupling and independence of $1 / \mathrm{f}$ noise in the central and autonomic nervous systems. Front Hum Neurosci 2014;8.

238 Roberge RJ, Kim J-H, Powell JB, et al. Impact of low filter resistances on subjective and physiological responses to filtering Facepiece respirators. PLoS One 2013;8:84901.

239 Liu Y, Ayaz H, Shewokis PA, et al. Multisubject "Learning” for Mental Workload Classification Using Concurrent EEG, fNIRS, and Physiological Measures. Front Hum Neurosci 2017;11.

240 Maddison R, Rawstorn JC, Rolleston A, et al. The remote exercise monitoring trial for exercise-based cardiac rehabilitation (REMOTE-CR): a randomised controlled trial protocol. BMC Public Health 2014;14.

241 Williamon A, Aufegger L, Wasley D, et al. Complexity of physiological responses decreases in high-stress musical performance. J. R. Soc. Interface 2013;10:20130719.

242 Ding S, Schumacher M, et al. Sensor monitoring of physical activity to improve glucose management in diabetic patients: a review. Sensors; $16: 589$.

243 Vergari F, Salmon Cinotti T, D'Elia A, et al. An integrated framework to achieve interoperability in person-centric health management. Int J Telemed Appl 2011;2011:1-10.
244 Greenwald R, Hayat MJ, Barton J, et al. A novel method for quantifying the inhaled dose of air pollutants based on heart rate, breathing rate and forced vital capacity. PLoS One 2016;11:e0147578.

245 Bachrach A, Fontbonne Y, Joufflineau C, et al. Audience entrainment during live contemporary dance performance: physiological and cognitive measures. Front Hum Neurosci 2015;9.

246 Mikita N, Hollocks MJ, Papadopoulos AS, et al. Irritability in boys with autism spectrum disorders: an investigation of physiological reactivity. J Child Psychol Psychiatr 2015;56:1118-26.

247 Sharma VKet al. Heart rate variability in adolescents normative data stratified by sex and physical activity. JCDR 2015;9.

248 Dhar P, Sharma VK, Hota KB, et al. Autonomic cardiovascular responses in acclimatized Lowlanders on prolonged stay at high altitude: a longitudinal follow up study. PLoS One 2014;9:84274.

249 Marinescu AC, Sharples S, Ritchie AC, et al. Physiological parameter response to variation of mental workload. Hum Factors 2018;60:31-56.

250 DiLeo T, Roberge RJ, Kim J-H. Effect of wearing an N95 filtering facepiece respirator on superomedial orbital infrared indirect brain temperature measurements. J Clin Monit Comput 2017;31:67-73.

251 Roberge RJ, Kim J-H, Powell JB. N95 respirator use during advanced pregnancy. Am J Infect Control 2014;42:1097100.

252 Villarejo M, Zapirain B, Zorrilla A. Algorithms based on CWT and classifiers to control cardiac alterations and stress using an ECG and a SCR. Sensors 2013;13:6141-70.

253 Dons E, Götschi T, Nieuwenhuijsen M, et al. Physical activity through sustainable transport approaches (pasta): protocol for a multi-centre, longitudinal study. BMC Public Health 2015;15:1126.

254 Engert V, Kok BE, Papassotiriou I, et al. Specific reduction in cortisol stress reactivity after social but not attention-based mental training. Sci Adv 2017;3:e1700495.

255 Pinti P, Merla A, Aichelburg C, et al. A novel GLM-based method for the automatic identification of functional events (AIDE) in fNIRS data recorded in naturalistic environments. Neuroimage 2017;155:291-304.

256 Sefton JM, McAdam JS, Pascoe DD, et al. Evaluation of 2 Heat-Mitigation methods in army trainees. J Athl Train 2016;51:936-45.

257 Triantafyllidis AK, Koutkias VG, Chouvarda I, et al. Framework of sensor-based monitoring for pervasive patient care. 2016;3:153-8.

258 Parate A, Chiu M-C, Chadowitz C, et al. RisQ: recognizing smoking gestures with inertial sensors on a Wristband. MobiSys 2014;2014:149-161.

259 Haller JM, Fehling PC, Barr DA, et al. Use of the HR index to predict maximal oxygen uptake during different exercise protocols. Physiol Rep 2013;1:124.

260 Natarajan A, Angarita G, Gaiser E, et al. Domain adaptation methods for improving Lab-to-field generalization of cocaine detection using wearable ECG. Proc ACM Int Conf Ubiquitous Comput 2016;2016:875-885.

261 Joufflineau C, Vincent C, Bachrach A. Synchronization, attention and transformation: multidimensional exploration of the aesthetic experience of contemporary dance spectators. Behav Sci 2018;8:24. 
262 Bobkowski W, Stefaniak ME, Krauze T, et al. Measures of heart rate variability in 24-h ECGs depend on age but not gender of healthy children. Front Physiol 2017;8:311.

263 Lai B, Rimmer J, Barstow B, et al. Teleexercise for persons with spinal cord injury: a mixed-methods feasibility case series. JMIR Rehabil Assist Technol;3:e8.

264 Leff DR, James DRC, Orihuela-Espina F, et al. The impact of expert visual guidance on trainee visual search strategy, visual attention and motor skills. Front Hum Neurosci 2015;9.

265 Andersen JP, Papazoglou K, Koskelainen M, et al. Applying resilience promotion training among special forces police officers. Sage Open 2015;5:215824401559044.

266 Stefani L, Maffulli N, Mascherini G, et al. Exercise as prescription therapy: benefits in cancer and hypertensive patients. Transl Med UniSa 2015;11.

267 Dolezal BA, Chudzynski JOY, Dickerson D, et al. Exercise training improves heart rate variability after methamphetamine dependency. Med Sci Sport Exerc 2014;46:1057-66.

268 ClinicalTrials.gov. Heart rate variability biofeedback for female sexual arousal disorder; 2016.

269 Lown M, Yue A, Lewith G, et al. Screening for Atrial Fibrillation using Economical and accurate TechnologY (SAFETY)—a pilot study. BMJ Open 2017;7:e013535.

270 Laxminarayan S, Rakesh V, Oyama T, et al. Individualized estimation of human core body temperature using noninvasive measurements. J Appl Physiol 2018;124:1387402.

271 Plews DJ, Scott B, Altini M, et al. Comparison of Heart-RateVariability recording with smartphone Photoplethysmography, polar H7 chest strap, and electrocardiography. Int J Sports Physiol Perform 2017;12:1324-8.

272 Cheatham SW, Kolber MJ, Ernst MP. Concurrent validity of resting pulse-rate measurements: a comparison of 2 smartphone applications, the polar $\mathrm{H} 7$ belt monitor, and a pulse oximeter with bluetooth. J Sport Rehabil 2015;24:1718.

273 Toennesen LL, Soerensen ED, Hostrup M, et al. Feasibility of high-intensity training in asthma. Eur Clin Respir J 2018;5:1468714.

274 Camacho-Cardenosa A, Camacho-Cardenosa M, Burtscher $\mathrm{M}$, et al. High-Intensity interval training in normobaric hypoxia leads to greater body fat loss in overweight/obese women than high-intensity interval training in normoxia. Front Physiol 2018;9:60.

275 Chisati EM, Vasseljen O. Aerobic endurance in HIV-positive young adults and HIV-negative controls in Malawi. Malawi Med J 2015;27:5.

276 Brouwers RWM, Kraal JJ, Traa SCJ, et al. Effects of cardiac telerehabilitation in patients with coronary artery disease using a personalised patient-centred web application: protocol for the SmartCare-CAD randomised controlled trial. BMC Cardiovasc Disord 2017;17.
277 Born D-P, Kunz P, Sperlich B. Reliability and validity of an agility-like incremental exercise test with multidirectional change-of-direction movements in response to a visual stimulus. Physiol Rep 2017;5:e13275.

278 Adams-Campbell LL, Dash C, Kim BH, et al. Cardiorespiratory fitness and metabolic syndrome in postmenopausal African-American women HHS public access. Int J Sport Med 2016;37:261-6.

279 Lukach AJ, Jedrziewski MK, Grove GA, et al. Rhythm experience and africana culture trial (react!): a culturally salient intervention to promote neurocognitive health, mood, and well-being in older African Americans. Contemp Clin Trials 2016;48:41-5.

280 Schoen JC, Machan JT, Dannecker M, et al. Team size and Stretching-Exercise effects on simulated chest compression performance and exertion. West J Emerg Med 2017;18:102534.

281 Prakash SKA, Tucker CS. Bounded Kalman filter method for motion-robust, non-contact heart rate estimation. Biomed Opt Express 2018;9:873-51.

282 Khundaqji H, Samain E, Climstein M, et al. A comparison of aerobic fitness testing on a swim bench and treadmill in a recreational surfing cohort: a pilot study. Sports 2018;6:54.

283 Nayak T, Zhang T, Mao Z, et al. Prediction of human performance using electroencephalography under different indoor room temperatures. Brain Sci;8:74.

284 Pontifex MB, Parks AC, Henning DA, et al. Single bouts of exercise selectively sustain attentional processes. Psychophysiology 2015;52:618-25.

285 Recinto C, Efthemeou T, Boffelli PT, et al. Effects of nasal or oral breathing on anaerobic power output and metabolic responses. Int J Exerc Sci 2017; 10:506-514.

286 Lupo C, Tessitore A, Gasperi L, et al. Session-RPE for quantifying the load of different youth basketball training sessions. Biol Sport 2017;34:11-17.

287 Berg vanden V, Saliasi E, de Groot RH, et al. Physical activity in the school setting: cognitive performance is not affected by three different types of acute exercise. Front Psychol 2016;7.

288 LY J, XL L, Liu Y, et al. Time-Dependent effects of acute exercise on university students' cognitive performance in temperate and cold environments. Front Psychol 2017;8.

289 Greenlee TA, Greene DR, Ward NJ, et al. Effectiveness of a 16-WEEK high-intensity CARDIORESISTANCE training program in adults. J Strength Cond Res 2017;31:2528-41.

290 Clemente FM, González-Víllora S, Delextrat A, et al. Effects of the sports level, format of the game and task condition on heart rate responses, technical and tactical performance of youth Basketball players. J Hum Kinet 2017;58:141-55.

291 ClinicalTrials.gov. Pulse oximeter test; 2017.

292 Welch J, Kanter B, Skora B, et al. Multi-Parameter vital sign database to assist in alarm optimization for general care units. J Clin Monit Comput 2016;30:895-900. 